\title{
Review
}

\section{Positive allosteric modulators to peptide GPCRs: a promising class of drugs}

\author{
Tamas BARTFAl ${ }^{1, *}$, Ming-wei WANG ${ }^{2}$ \\ ${ }^{1}$ Department of Chemical Physiology, The Scripps Research Institute, La Jolla, CA 92037, USA; ${ }^{2}$ The National Center for Drug Screen- \\ ing and the CAS Key Laboratory of Receptor Research, Shanghai Institute of Materia Medica, Chinese Academy of Sciences (CAS), \\ Shanghai 201203, China
}

\begin{abstract}
The task of finding selective and stable peptide receptor agonists with low molecular weight, desirable pharmacokinetic properties and penetrable to the blood-brain barrier has proven too difficult for many highly coveted drug targets, including receptors for endothelin, vasoactive intestinal peptide and galanin. These receptors and ligand-gated ion channels activated by structurally simple agonists such as glutamate, glycine and GABA present such a narrow chemical space that the design of subtype-selective molecules capable of distinguishing a dozen of glutamate and GABA receptor subtypes and possessing desirable pharmacokinetic properties has also been problematic. In contrast, the pharmaceutical industry demonstrates a remarkable success in developing 1,4-benzodiazepines, positive allosteric modulators (PMAs) of the $\mathrm{GABA}_{\mathrm{A}}$ receptor. They were synthesized over 50 years ago and discovered to have anxiolytic potential through an in vivo assay. As exemplified by Librium, Valium and Dormicum, these allosteric ligands of the receptor became the world's first blockbuster drugs. Through molecular manipulation over the past 2 decades, including mutations and knockouts of the endogenous ligands or their receptors, and by in-depth physiological and pharmacological studies, more peptide and glutamate receptors have become well-validated drug targets for which an agonist is sought. In such cases, the pursuit for PAMs has also intensified, and a working paradigm to identify drug candidates that are designed as PAMs has emerged. This review, which focuses on the general principles of finding PAMs of peptide receptors in the 21st century, describes the workflow and some of its resulting compounds such as PAMs of galanin receptor 2 that act as potent anticonvulsant agents.
\end{abstract}

Keywords: neuropeptide; galanin; GPCR; allosteric mudulation; CYM2503; anticonvulsant; drug screening

Acta Pharmacologica Sinica (2013) 34: 880-885; doi: 10.1038/aps.2013.20; published online 29 Apr 2013

\section{Introduction}

Allosteric modulators of proteins are well known. They interact with a site other than the binding site of the enzyme substrate (allosteric). In the case of many biosynthetic enzymes, their characterization was pioneered by Changeux and Pardee $^{[1-7]}$, who investigated where do the molecules (eg, feedback inhibitors of amino acid) bind and how does nucleotide biosynthesis on the enzyme catalyze the first step in the cascade $^{[4]}$. The key examples of allosteric modulator characterization are the detailed studies on aspartic transcarbamylase and its feedback inhibition ${ }^{[4,5]}$. Other examples of allosteric actions involve non-enzymatic proteins such as the oxygen carrier hemoglobin, whose affinity is regulated by protons through the Bohr effect without involving binding to the $\mathrm{O}_{2}$ site, or by 2,3-bisphosphoglycerate binding to an allosteric site on hemo-

\footnotetext{
* To whom correspondence should be addressed.

E-mail tbartfai@scripps.edu

Received 2012-12-03 Accepted 2013-02-07
}

globin that affects $\mathrm{O}_{2}$ trapping ${ }^{[8,9]}$.

Pharmacological uses of receptor allosteric modulators The pharmacological utilization of allosteric sites was either unconscious or unplanned, because endogenous ligand binding to orthosteric sites or the receptor itself was not defined in molecular terms. When drug molecules were tested in functional assays, the molecular identity of the binding site was not questioned and determined: only functional changes were measured. Thus, it did not matter for the functionality "black box" assay whether the drug molecule is an agonist binding to the orthosteric site of a receptor where endogenous ligand also binds, or it interacts with an allosteric site and acts as a positive allosteric modulator (PAM). When Leo Sternbach ${ }^{[10,11]}$ synthesized Valium, a 1,4-benzodiazepine, the molecule was tested in vivo in a rodent anxiety model. Due to the fact that it was highly efficacious and acutely safe, it never mattered if Valium binds to an allosteric site on one subunit of the heteropentameric $\mathrm{GABA}_{\mathrm{A}}$ receptor or to its coupled chloride channel. 
Today, allosteric ligands are also introduced for other pentameric ligand-gated ion channels such as a7 nicotinic acetylcholine receptor ${ }^{[12]}$ and G-protein coupled receptors (GPCRs). The latter represent the largest class of cell surface proteins and the major receptor class for neuropeptides including GABA receptors $^{[13-15]}$.

In today's target-based drug discovery, a protein molecule, $e g$, a peptide receptor, is identified as a drug target based on the physiological and pathophysiological effects caused by mutations in its agonist or in the receptor molecule per $s e^{[14,15]}$. We are constantly searching for selective, high-affinity ligands to these putative drug targets via high-throughput molecular and cellular screening assays (HTS), which can be designed to identify ligands that bind either to the orthosteric or allosteric site of a receptor ${ }^{[16,17]}$.

The pharmacological application of metabotropic glutamate receptors (mGluRs) and glutamate-activated ion channels, such as NMDA and AMPA receptors, is rather limited by the fact that the endogenous ligand glutamate is a chemically simple molecule: its binding site (the orthosteric site) occupies a very narrow chemical space for the design of receptor subtype-selective exogenous agonists, although such compounds, eg, the mGluR2/3 agonist, could serve as an anti-psychotic agent ${ }^{[18]}$. It became clear to drug developers at Roche ${ }^{[19-21]}$, Merck $^{[22,23]}$ and later in the entire industry ${ }^{[24]}$ that if there were allosteric sites on these receptors, they might hold promise. Drugs that bind to these sites would only be effective when an endogenous agonist occupies the orthosteric site, meaning that the biological rhythm of agonist action is dictated by factors that regulate the release of the endogenous ligand. The allosteric molecules that enhanced agonist action, when identified, have proven to be safe and caused no desensitization, a feature different from directly acting agonists in the monoaminergic system or of NK1 receptors.

\section{Screening for receptor allosteric modulators}

The problem in identifying allosteric modulators has been that there are no inferences about the chemical structure of allosteric modulators. Until one or a few allosteric modulators, which bind in the presence of the endogenous ligand or an agonist that occupies the orthosteric site, are attributed to a given receptor, even the existence of an allosteric binding site is in question. Thus, diverse chemical libraries are required to discover an initial allosteric modulator. At the beginning, this approach led to serious disagreements within the community between "screening groups" and biologists about whether it is worth the effort to perform such screens and how to do them. Today, with several dozens of PAMs and negative allosteric modulators (NAMs) in clinical trials as a result of focused efforts, such discussions are largely over. X-ray structures of several types of receptors, including one of the most commonly used drug targets, GPCRs, also strongly suggest the presence of allosteric sites on monomeric, heterodimeric and homodimeric GPCRs ${ }^{[25-27]}$.

It is understood from the experience with numerous early screens that cellular assays where receptor occupancy by an orthosteric agonist can be measured are best suited to identify allosteric modulators. One needs to use a stable agonist in concentrations ranging between $20 \%$ and $30 \%$ of the maximal effect in the screening efforts $\left(\mathrm{EC}_{20}\right.$ to $\left.\mathrm{EC}_{30}\right)$. Clearly, there is a room for identification of PAMs, while NAMs could be screened in the same setting using an orthosteric agonist at $\mathrm{EC}_{80}$ (Boxes 1 and 2). The work that discovered CYM2503 ${ }^{[28]}$ as a PAM of galanin receptor type $2^{[29]}$ is described below in detail.

Box 1. Approaches to identifying drugs acting at peptide receptors.

\section{Degradation inhibitors}

- Identify the degradation sites and inhibit the specific peptidases (ie, raise half-life of the endogenous ligand): glucagon-like peptide- 1 and DPP-4 inhibitors.

- Make peptidomimetics that do not have fissile peptide bonds sensitive to these peptidases.

2. Agonist molecules and/or molecules acting at the receptor

- Active fragments are identified.

- L-Ala substitution to identify pharmacophores.

- Low molecule weight, nonpeptidic agonists: based on the information regarding pharmacophore side chains and their distances; the side chains are displayed on scaffolds providing the distances.

- Synthetic peptide libraries are screened.

- Positive allosteric modulators (PAMs) are screened.

Box 2. How to find allosteric ligands to peptide receptors systematically.

1. Generate a (peptidolitically) stable (even if it is low affinity, non subtype selective) agonist that occupies the ortosteric site (the site occupied by the endogenous ligand).

2. Conduct cellular screens at $\mathbf{2 0} \%$ and $\mathbf{8 0} \%$ receptor occupancy using the "stable agonist" above; this gives the best chance to get a signal for positive and negative allosteric modulators.

For peptide receptor PAMs, the biggest problem is to find a peptidolitically stable orthosteric agonist - the tool compounds (like galnon or galmic for GALR2, and Boc5 for glucagon-like peptide-1 receptor).

\section{Efforts to find peptide receptor agonists: as exemplified for the search for galanin receptor agonists}

Galanin is a 29 amino acid (aa) C-terminally amidated neuropeptide in pigs and rodents and a 30 aa long neuropeptide in humans ${ }^{[30-33]}$. It reacts with three types of GPCRs (galanin receptors 1-3, GALR1-3) (Figure 1) and GALR1 and GALR2 are involved in anticonvulsant activity $^{[33,34]}$.

1) The pharmacophores and the orthosteric site of GALR1: The pharmacophores involved in the interaction with the receptor - ie, with its orthosteric site - were determined by two mutually complementary strategies: L-Ala substitution of the amino acids in galanin ${ }^{[35]}$ and site-directed mutagenesis of the galanin receptor residues suggested by modeling the 


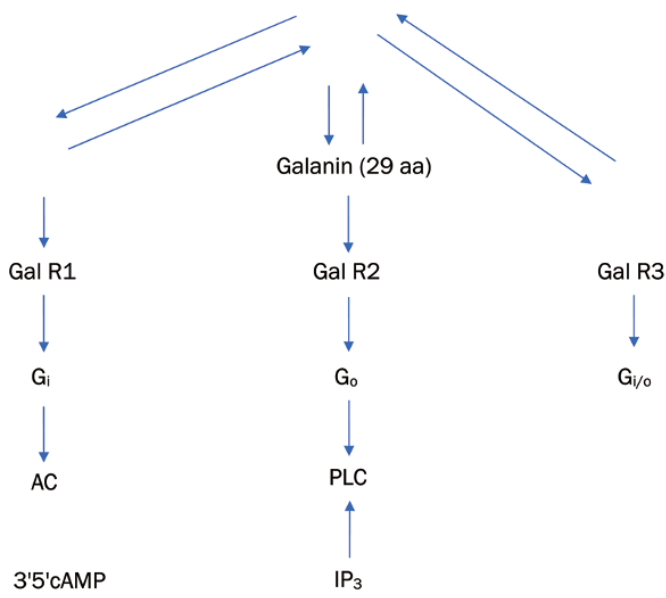

Four million compounds were screened by Roche, Merck, Pfizer, and Johnson \& Johnson, but neither agonist nor antagonist was found at $10 \mu \mathrm{mol} / \mathrm{L}$ cut-off. We had to start to look for allosteric ligands but galanin had a half-life of only $2.5 \mathrm{~min}$ in the cellular assay, so at $20 \%$ occupancy the results were extremely variable and PAMs were missed. We needed a peptidolitically stable galanin receptor agonist (not necessarily subtype selective).

Figure 1. The neuropeptide galanin binds to three G-protein coupled receptors (GPCRs) with $\mathrm{K}_{\mathrm{d}}$ of $1 \mathrm{nmol} / \mathrm{L}$ in the brain and spinal cord. PAM, positive allosteric modulator; aa, amino acid.

agonist-receptor interactions ${ }^{[36]}$. These studies showed that the residues Trp 2, Asn 5, and Tyr 9 at the N-terminus of galanin (Figure 2A) were essential for binding to the surface residues of the galanin receptor, including His 264, His 267, Glu 271, Phe 115, and Phe 282 that constitute the orthosteric binding site $^{[37]}$. Binding of galanin-29 or -30 to the native receptor occurs with high affinity $\left(\mathrm{K}_{\mathrm{d}} 0.3-1 \mathrm{nmol} / \mathrm{L}\right)$ and high selectivity. It was also demonstrated that the shortest peptide, galanin 1-9, despite having all the pharmacophores, has a low affinity $(\mu \mathrm{mol} / \mathrm{L})$ and is peptidolytically unstable. The longer galanin 1-16 peptide has greater stability (half-life: $2-5 \mathrm{~min}$ in the circulation) and was employed in some studies, but it does not penetrate the blood-brain barrier $(\mathrm{BBB})^{[38]}$. In addition, galanin 3-29 does not act as an agonist ${ }^{[39]}$, implying that inhibition of the dipeptidyl peptidase that cut the 1-2 residues (Gly-Trp) of galanin may augment the action of endogenous galanin. However, the attempt to find selective inhibitors of the dipeptidyl peptidase failed (Box 3).

2) Search for non-peptidic agonists: The distance between the two most distal pharmacophores, the N-terminal primary amino group and Tyr 9, is large for a small molecule scaffold to maintain. Nevertheless, peptides from a peptide library based on the pharmacophores were tested, and galnon was identified as a low molecular weight, systemically active galanin receptor agonist (Figure 2). It has a $K_{d}$ of $1 \mu \mathrm{mol} / \mathrm{L}$, exhibits no selectivity among the three galanin receptor subtypes, but is peptidolytically stable with a half-life of approximately 60 minutes and crosses the $\mathrm{BBB}^{[40]}$. This compound exhibited a remarkable and dose-dependent efficacy as an anticonvulsant,

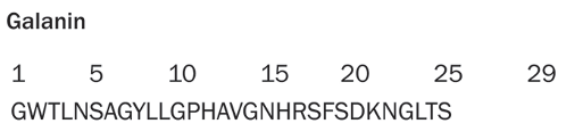<smiles>Cc1cc(=O)oc2cc(NC(=O)C(CCCCN)NC(=O)C(CC3CCCCC3)NC(=O)OCC3c4ccccc4-c4ccccc43)ccc12</smiles>

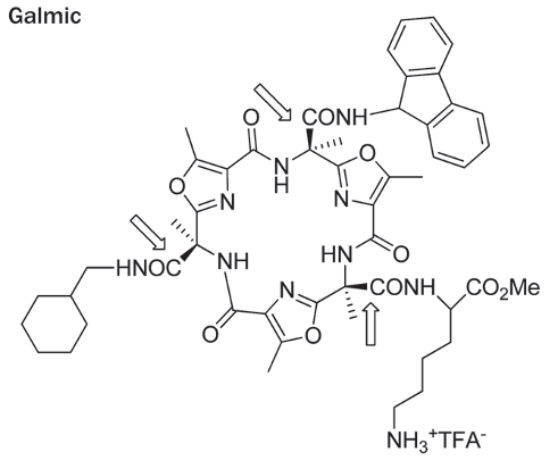

CYM2503

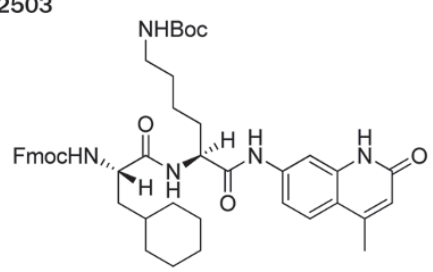

Figure 2. Galanin receptor agonists and positive allosteric modulator. Galanin is the endogenous peptide ligand, not receptor subtype selective. Galnon is a nonpeptidic, systemically active low affinity orthosteric agonist that is not receptor subtype selective. Galmic is a nonpeptidic, systemically active low affinity orthosteric agonist that is not receptor subtype selective. CYM2503 is a systemically active, positive allosteric modulator to the GALR2 receptor.

and thus reinvigorated the search for low molecular weight agonists and later for PAMs of the galanin receptor.

The pharmacophores were displayed on a cyclic scaffold by the Rebek group ${ }^{[41]}$, providing compound galmic (Figure 2). Galmic has a $K_{d}$ of 1-2 $\mu \mathrm{mol} / \mathrm{L}$, possesses no receptor subtype selectivity, is peptidolytically stable with a half-life of approximately $120 \mathrm{~min}$ and crosses the BBB. This compound, like galnon, showed a dose-dependent and strong efficacy in several seizure models ${ }^{[42]}$.

Both galnon and galmic are capable of competing with galanin and could displace the endogenous peptide agonist from the orthosteric site. Their $\mathrm{K}_{\mathrm{d}}$ and $\mathrm{EC}_{50}$ values correspond closely. Attempts to improve their affinities or to endow them with receptor subtype selectivity have not been pursued after the first series of SAR studies showed very narrow space for 
Box 3. Workflow leading to discovery of galanin receptor agonists or positive allosteric modulators for therapy of seizures and neuropathic pain.

- Degradation of galanin is catalyzed by broad specificity diaminopeptidyl peptidases, which are too non-specific targets to utilize pharmacologically.

- Active fragment is identified: $\mathrm{N}$-terminal $1-16$ galanin is a full agonist in feeding, seizure and pain models (1992).

- L-Ala substitution to identify the pharmacophores: $\mathrm{N}$-terminal $\mathrm{NH}_{2}$ group, Trp 2, Asn 5, and Tyr 9 are all required for high affinity agonist action (1996).

- Low molecular weight, nonpeptidic agonists: based on the information regarding the pharmacophores, synthetic peptide libraries of Gly/Ala/ Leu, Phe/Tyr/Trp, Asp/Asn, and Phe/Tyr/Trp amide were screened, and galnon was found. It behaves as a systemically active GALR1 and GALR2 agonist with an $\mathrm{EC}_{50}$ of $1 \mu \mathrm{mol} / \mathrm{L}$ (2002).

- Based on the information of the pharmacophores, the side chains are displayed on scaffolds providing the distances. Galmic was synthesized which behaves as a systemically active GALR1 and GALR2 agonist with an $\mathrm{EC}_{50}$ of $1 \mu \mathrm{mol} / \mathrm{L}$ (2004).

- Positive allosteric modulators are screened using galanin $\mathrm{EC}_{10}$, galnon $\mathrm{EC}_{30}$ or galmic $\mathrm{EC}_{30}$ on a cell line heterologously expressing GALR1, GALR2, or both. CYM2053 is as potent anticonvulsant as three last approved drugs and it is also active in limbic seizures (2010).

modifications. The desired affinity and pharmacokinetic properties for an orthosteric agonist of a central peptide receptor could not be reached with these compounds or close analogs of them. Other efforts to use the orthosteric site of galanin receptors are based on fatty acid derivatives of the peptide to enhance its permeability, to create a systemically active compound of the polar, short-lived neuropeptide, and to retain its pharmacophores ${ }^{[43-45]}$.

\section{Search for PAMs of galanin receptors}

The parallel development of multiple cellular assays in highthroughput format to search for GPCR ligands (agonists and antagonists) has provided us with the tools to discover PAMs. A general working paradigm of these efforts is summarized in Box 2. The advantages of PAMs over full agonists in binding to the orthosteric site are numerous. As discussed in the introduction, they are unlikely to cause receptor desensitization as some agonists do, they preserve the biological rhythm of the agonist action because they are only active when the endogenous agonist is released, and they can be of a completely different chemical structure as opposed to orthosteric site binders so that small molecules with desirable pharmacokinetic properties may be easier to find. However, there are two major concerns: the existence of a relatively stable orthosteric agonist to carry out the HTS assay reproducibly and lack of prior clues about which compound library to screen and to what structural classes these PAMs will belong. The access to two nonpeptidic, peptidolytically stable full agonists galnon ${ }^{[40]}$ and galmic ${ }^{[41]}$ that bind to the orthosteric site of GALR1 and
GALR2 has solved the first concern above, although they are not of high-affinity or subtype-selective but can be applied at $\mathrm{EC}_{20}$ and $\mathrm{EC}_{80}$ concentrations (Box 3) to search for PAMs and NAMs in HTS using cell lines expressing GALR1 or GALR2 receptors.

In the case of the GALR2 receptor, IP3 production was monitored as the signal (Figure 1), a diverse chemical library was screened, and the hits were optimized to yield a PAM CYM2503 (Figure 2). This compound does not displace ${ }^{125} \mathrm{I}-$ Tyr 9 galanin 1-29 from the orthosteric site (as galnon and galmic do), so it is a bona fide allosteric ligand. It enhances IP3 production elicited by sub-maximal concentrations of the orthosteric agonists galanin, galnon and galmic. Thus, CYM2503 is a PAM with receptor subtype selectivity for GALR2 only ${ }^{[28]}$.

The effects of CYM2503 are remarkably potent in the electroconvulsive shock and status epilepticus tests in vivo (Figure $3)$, where it was found to be as potent in reducing mortality in mice as the golden standard in the clinic, Keppra ${ }^{\circledR}$ (Levetiracetam). The animal experimentation data are sufficiently promising for initiating formulation work on this compound to improve its pharmacokinetic properties.

A Lowered mortality in status epilepticus Li-pilocarpine model

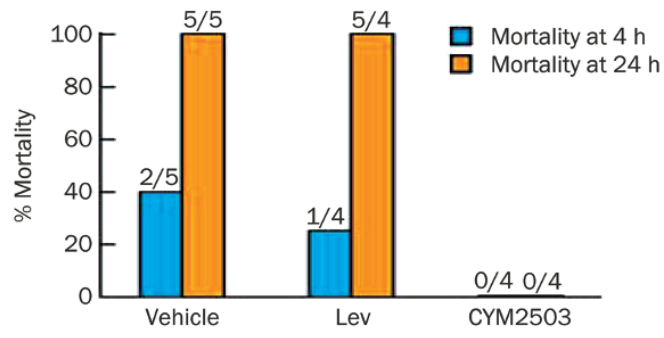

B Lowered mortality in electroshock induced seizure model

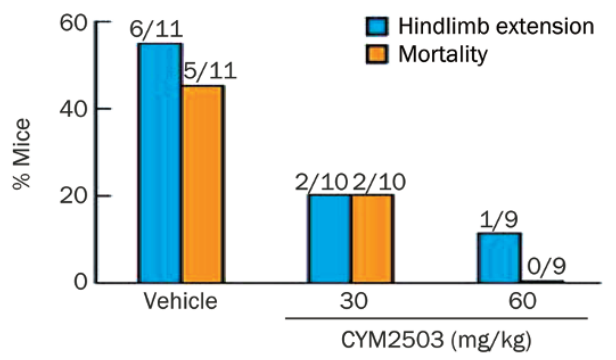

Figure 3. CYM2503 lowers mortality in seizure models. (A) Lowered mortality in the Li-pilocarpine model of status epilepticus. (B) Lowered mortality in an electroshock-induced seizure model.

\section{Conclusions}

Since the first design-based, allosteric modulators for mGLuRs and $\mathrm{GABA}_{\mathrm{A}}$ receptors were developed approximately 10 years ago, we have acquired experience in identifying new members of this class of drugs. These practices confirmed that designbased allosteric modulators are a particularly attractive drug 
class when peptide receptor agonists are of low molecular weight and when derivation of the peptide fused with human serum albumin or with $F_{c}$ antibody fragment, etc. is not applicable, as in the case of the peripherally active glucagonlike peptide- 1 analogs used in diabetes therapy ${ }^{[46,47]}$. For glutamate, glycine and GABA receptors in the brain, we will see continued development of allosteric modulators of low molecular weight that can penetrate the BBB. Insights gained from recent advances in structural biology with availability of a dozen of ligand-bound GPCR X-ray structures will certainly accelerate these developments.

\section{Acknowledgements}

This manuscript is based on collaborations with Ulo LANGEL, Ed ROBERTS, Julius REBEK and Xiaoying LU. Several in vivo assays were carried out in other laboratories. We are most grateful to Claude WASTERLAIN, to the NINCDS for screening of anticonvulsants, and to Dale E MAIS for critical review of this manuscript.

\section{References}

1 Changeux JP. Allosteric proteins: from regulatory enzymes to receptors-personal recollections. Bioessays 1993; 15: 625-34.

2 Changeux JP. 50th anniversary of the word "allosteric". Protein Sci 2011; 20: 1119-24.

3 Changeux JP. Allostery and the Monod-Wyman-Changeux model after 50 years. Annu Rev Biophys 2012; 41: 103-33.

4 Changeux JP, Gerhart JC, Schachman HK. Allosteric interactions in aspartate transcarbamylase. I. Binding of specific ligands to the native enzyme and its isolated subunits. Biochemistry 1968; 7 : 531-8.

5 Changeux JP, Rubin MM. Allosteric interactions in aspartate transcarbamylase. 3. Interpretation of experimental data in terms of the model of Monod, Wyman, and Changeux. Biochemistry 1968; 7: 553-61.

6 Monod J, Wyman J, Changeux JP. On the nature of allosteric transitions: a plausible model. J Mol Biol 1965; 12: 88-118.

7 Rubin MM, Changeux JP. On the nature of allosteric transitions: implications of non-exclusive ligand binding. J Mol Biol 1966; 21 : 265-74.

8 Bellelli A, Brunori M. Hemoglobin allostery: variations on the theme. Biochim Biophys Acta 2011; 1807: 1262-72.

9 Fang TY, Zou M, Simplaceanu V, Ho NT, Ho C. Assessment of roles of surface histidyl residues in the molecular basis of the Bohr effect and of beta 143 histidine in the binding of 2,3-bisphosphoglycerate in human normal adult hemoglobin. Biochemistry 1999; 38: 1342332.

10 Sternbach LH. The discovery of librium. Agents Actions 1972; 2: 193-6.

11 Sternbach LH. 1,4-benzodiazepines. Chemistry and some aspects of the structure-activity relationship. Angew Chem Int Ed Engl 1971; 10 : 34-43.

12 Krause RM, Buisson B, Bertrand S, Corringer PJ, Galzi JL, Changeux $J P$, et al. Ivermectin: a positive allosteric effector of the $\alpha 7$ neuronal nicotinic acetylcholine receptor. Mol Pharmacol 1998; 53: 283-94.

13 Urwyler S. Allosteric modulation of family C G-protein-coupled receptors: from molecular insights to therapeutic perspectives. Pharmacol Rev 2011; 63: 59-126.

14 Hoyer D, Bartfai T. Neuropeptides and neuropeptide receptors: drug targets, and peptide and non-peptide ligands: a tribute to professor Dieter Seebach. Chem Biodivers 2012; 9: 2367-87.

15 McColl CD, Jacoby AS, Shine J, lismaa TP, Bekkers JM. Galanin receptor-1 knockout mice exhibit spontaneous epilepsy, abnormal EEGs and altered inhibition in the hippocampus. Neuropharmacology 2006; 50: 209-18.

16 Kenakin T, Miller LJ. Seven transmembrane receptors as shapeshifting proteins: the impact of allosteric modulation and functional selectivity on new drug discovery. Pharmacol Rev 2010; 62: 265-304.

17 Kenakin T. Functional selectivity in GPCR modulator screening. Comb Chem High Throughput Screen 2008; 11: 337-43.

18 Govek SP, Bonnefous C, Hutchinson JH, Kamenecka T, McQuiston J, Pracitto R, et al. Benzazoles as allosteric potentiators of metabotropic glutamate receptor 2 (mGluR2): efficacy in an animal model for schizophrenia. Bioorg Med Chem Lett 2005; 15: 4068-72.

19 Kew JN. Positive and negative allosteric modulation of metabotropic glutamate receptors: emerging therapeutic potential. Pharmacol Ther 2004; 104: 233-44.

20 Kew JN, Kemp JA. Ionotropic and metabotropic glutamate receptor structure and pharmacology. Psychopharmacology (Berl) 2005; 179: 4-29.

21 Knoflach F, Mutel V, Jolidon S, Kew JN, Malherbe P, Vieira E, et al. Positive allosteric modulators of metabotropic glutamate 1 receptor: characterization, mechanism of action, and binding site. Proc Natl Acad Sci U S A 2001; 98: 13402-7.

22 Gregory KJ, Dong EN, Meiler J, Conn PJ. Allosteric modulation of metabotropic glutamate receptors: structural insights and therapeutic potential. Neuropharmacology 2011; 60: 66-81.

23 Marino MJ, Williams DL Jr, O’Brien JA, Valenti O, McDonald TP, Clements MK, et al. Allosteric modulation of group III metabotropic glutamate receptor 4: a potential approach to Parkinson's disease treatment. Proc Natl Acad Sci U S A 2003; 100: 13668-73.

24 Adams CL, Lawrence AJ. CGP7930: a positive allosteric modulator of the GABAB receptor. CNS Drug Rev 2007; 13: 308-16.

25 Shoichet BK, Kobilka BK. Structure-based drug screening for G-protein-coupled receptors. Trends Pharmacol Sci 2012; 33: 268-72.

26 Rosenbaum DM, Rasmussen SG, Kobilka BK. The structure and function of G-protein-coupled receptors. Nature 2009; 459: 356-63.

27 Kirkpatrick A, Heo J, Abrol R, Goddard WA, 3rd. Predicted structure of agonist-bound glucagon-like peptide 1 receptor, a class B G proteincoupled receptor. Proc Natl Acad Sci U S A 2012; 109: 19988-93.

28 Lu X, Roberts E, Xia F, Sanchez-Alavez M, Liu T, Baldwin R, et al. GalR2-positive allosteric modulator exhibits anticonvulsant effects in animal models. Proc Natl Acad Sci U S A 2010; 107: 15229-34.

29 O'Donnell D, Ahmad S, Wahlestedt C, Walker P. Expression of the novel galanin receptor subtype GALR2 in the adult rat CNS: distinct distribution from GALR1. J Comp Neurol 1999; 409: 469-81.

30 Bloomquist BT, Beauchamp MR, Zhelnin L, Brown SE, Gore-Willse AR, Gregor P, et al. Cloning and expression of the human galanin receptor GaIR2. Biochem Biophys Res Commun 1998; 243: 474-9.

31 Mitsukawa K, Lu X, Bartfai T. Galanin, galanin receptors, and drug targets. Exs 2010; 102: 7-23.

32 Mazarati A, Lundstrom L, Sollenberg U, Shin D, Langel U, Sankar R. Regulation of kindling epileptogenesis by hippocampal galanin type 1 and type 2 receptors: The effects of subtype-selective agonists and the role of G-protein-mediated signaling. J Pharmacol Exp Ther 2006; 318: $700-8$

33 Lerner JT, Sankar R, Mazarati AM. Galanin and epilepsy. EXS 2010; 102: 183-94. 
34 Kapur J. Galanin receptors modulate seizures. Epilepsy Curr 2011; 11: $125-7$.

35 Land T, Langel U, Low M, Berthold M, Unden A, Bartfai T. Linear and cyclic $\mathrm{N}$-terminal galanin fragments and analogs as ligands at the hypothalamic galanin receptor. Int J Pept Protein Res 1991; 38 : 267-72.

36 Kask K, Berthold M, Kahl U, Nordvall G, Bartfai T. Delineation of the peptide binding site of the human galanin receptor. EMBO J 1996; 15: 236-44.

37 Kask K, Berthold M, Kahl U, Jureus A, Nordvall G, Langel U, et al. Mutagenesis study on human galanin receptor GalR1 reveals domains involved in ligand binding. Ann NY Acad Sci 1998; 863: 78-85.

38 Fisone G, Berthold M, Bedecs K, Unden A, Bartfai T, Bertorelli R, et al. $\mathrm{N}$-terminal galanin-(1-16) fragment is an agonist at the hippocampal galanin receptor. Proc Natl Acad Sci U S A 1989; 86: 9588-91.

39 Land T, Langel U, Bartfai T. Hypothalamic degradation of galanin (1-29) and galanin (1-16): identification and characterization of the peptidolytic products. Brain Res 1991; 558: 245-50.

40 Saar K, Mazarati AM, Mahlapuu R, Hallnemo G, Soomets U, Kilk K, et al. Anticonvulsant activity of a nonpeptide galanin receptor agonist. Proc Natl Acad Sci U S A 2002; 99: 7136-41.

41 Ceide SC, Trembleau L, Haberhauer G, Somogyi L, Lu X, Bartfai T, et al. Synthesis of galmic: a nonpeptide galanin receptor agonist. Proc
Natl Acad Sci U S A 2004; 101: 16727-32.

42 Mazarati A, Lu X. Regulation of limbic status epilepticus by hippocampal galanin type 1 and type 2 receptors. Neuropeptides 2005; 39: 277-80.

43 Robertson CR, Pruess TH, Grussendorf E, White HS, Bulaj G. Generating orally active galanin analogues with analgesic activities. ChemMedChem 2012; 7: 903-9.

44 Bulaj G, Green BR, Lee HK, Robertson CR, White K, Zhang L, et al. Design, synthesis, and characterization of high-affinity, systemicallyactive galanin analogues with potent anticonvulsant activities. J Med Chem 2008; 51: 8038-47.

45 Parthiban M, Shanmughavel P. Three dimensional modeling of $\mathrm{N}$-terminal region of galanin and its interaction with the galanin receptor. Bioinformation 2007; 2: 119-25.

46 Leger R, Thibaudeau K, Robitaille M, Quraishi O, van Wyk P, BousquetGagnon N, et al. Identification of CJC-1131-albumin bioconjugate as a stable and bioactive GLP-1 (7-36) analog. Bioorg Med Chem Lett 2004; 14: 4395-8.

47 Jimenez-Solem E, Rasmussen MH, Christensen M, Knop FK. Dulaglutide, a long-acting GLP-1 analog fused with an Fc antibody fragment for the potential treatment of type 2 diabetes. Curr Opin Mol Ther 2010; 12: 790-7. 